\title{
High myopia is not associated with single nucleotide polymorphisms in the COL2A1 gene in the Chinese population
}

\author{
JUAN WANG, PANFENG WANG, YANG GAO, SHIQIANG LI, \\ XUESHAN XIAO and QINGJIONG ZHANG \\ State Key Laboratory of Ophthalmology, Zhongshan Ophthalmic Center, \\ Sun Yat-sen University, Guangzhou, Guangdong 510060, P.R. China
}

Received July 8, 2011; Accepted October 4, 2011

DOI: $10.3892 / \mathrm{mmr} .2011 .626$

\begin{abstract}
Association studies have revealed that the rs1635529 polymorphism in the COL2Al (collagen, type II, $\alpha 1$ ) gene may be a potential candidate for myopia development and may be associated with myopia in Caucasians. The aim of this study was to investigate the association of high myopia with 5 polymorphisms including rs1635529 in COL2Al in a Chinese population. Genomic DNA samples were selected from our Genomic DNA Repository for Myopia. A total of 581 high myopia individuals (refraction $\leq-6.00 \mathrm{D}$, spherical equivalent) and 384 normal controls (refraction between $-0.50 \mathrm{D}$ and $+2.00 \mathrm{D}$, spherical equivalent) from a Han Chinese cohort participated in the association study. Allelic discrimination assay was performed on five single nucleotide polymorphisms (SNPs) (rs1635529, rs60542319, rs1635530, rs1635531 and rs954326) in COL2A 1 by direct sequence analysis of polymerase chain reaction products. The genotype, allele and haplotype frequencies of the SNPs between cases and controls were compared by Chi-square test for association analysis. Based on the results, SNPs at rs1635530 and rs1635531 were found to be monomorphic in all subjects. There was no statistically significant difference in genotype, allele and haplotype frequencies for the other three SNPs (rs1635529, rs60542319 and rs954326) between the high myopia group and the control group. We do not find evidence to support an association of SNP rs1635529 in COL2Al with high myopia in the Chinese population studied, nor of the other two SNPs (rs60542319 and rs954326).
\end{abstract}

\section{Introduction}

The global prevalence of refractive errors has been estimated from 800 million to 2.3 billion (1). Myopia is an increasingly

Correspondence to: Dr Qingjiong Zhang, Ophthalmic Genetics and Molecular Biology, Zhongshan Ophthalmic Center, Sun Yat-sen University, 54 Xianlie Road, Guangzhou, Guangdong 510060, P.R. China

E-mail: zhangqji@mail.sysu.edu.cn; qingjiongzhang@yahoo.com

Key words: COL2A1, single nucleotide polymorphism, myopia, association, Chinese population crucial global public health issue (2-4). The prevalence of myopia has been reported as high as $70-90 \%$ in certain Asian countries, 30-40\% in Europe and the US, and 10-20\% in Africa $(3,5-10)$. Its extreme form, high myopia, is the fourth most common cause of irreversible blindness in the world $(11,12)$.

Myopia is a common, complex and multifactorial disorder. More and more research into genetic factors of myopia give rise to its importance, with improved understanding of its biological mechanism $(2,13-18)$. The association study of candidate genes and loci has been evaluated and the available evidence suggests the involvement of certain genes and loci in myopia in different ethnic populations (19-26).

The COL2A1 gene (OMIM120140), mapping to $12 \mathrm{q} 13.11$, encodes the $\alpha-1$ chain of type II collagen, a fibrillar collagen widely expressed in cartilage and the vitreous humor of the eye. COL2A1 mutations are reported to cause Stickler syndrome type I (OMIM 108300) (27), with severe myopia as a consistent phenotype. Recently, a significant association was identified between SNP rs1635529 located in the COL2A1 locus and myopia in Caucasian family-based association studies in two separate reports $(23,28)$.

We used a case-control association study approach to conduct candidate genetic analysis to investigate the association between high myopia and SNP markers in intron 1 of the COL2A1 gene (rs1635529 and a number of adjacent SNPs). It is the first report of a COL2A1 SNP site association study in a large Han Chinese cohort

\section{Materials and methods}

Subjects. In total, 965 unrelated individuals participated in this study, including 581 high myopia cases (sphere at each meridian $\leq-6.00 \mathrm{D}$ ), and 384 university students without myopia (spherical equivalent between -0.50 and $+2.00 \mathrm{D}$ ) were used as normal controls.

Refractive errors were measured by an autorefractor (Topcon KR-8000, Paramus, NJ, USA) after mydriasis with compound tropicamide (Mydrin ${ }^{\circledR}$-P; Santen Pharmaceutical Co. Ltd., Osaka, Japan). All cases and controls received an ocular biometry examination using IOL master V5 (Carl Zeiss Meditec AG, Jena, Germany). Written informed consent, conforming to the tenets of the Declaration of Helsinki, was obtained from each participant prior to the study. This study 
Table I. Primers for amplifying the fragment encompassing the five single nucleotide polymorphisms of interest.

Primer sequence Product length Annealing temperature

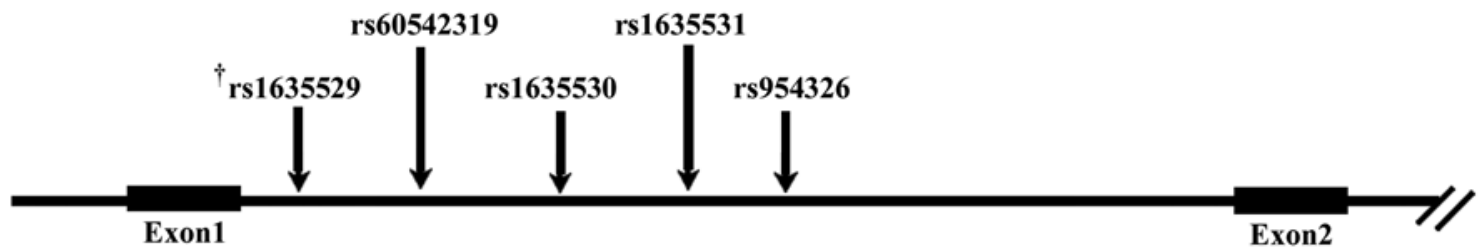

Figure 1. Schematic representation of five SNPs (rs1635529, rs60542319, rs1635530, rs1635531 and rs954326) showing their location on the COL2A1 gene (not to scale). In the present study, all five SNPs were selected for COL2A1. Vertical arrows show the SNPs with their rs numbers. Black boxes indicate the position of exons (based on NM_001844.4, Genebank). 'Single nucleotide polymorphism associated with myopia as reported in a Caucasian population..

A

rs1635529

A/A
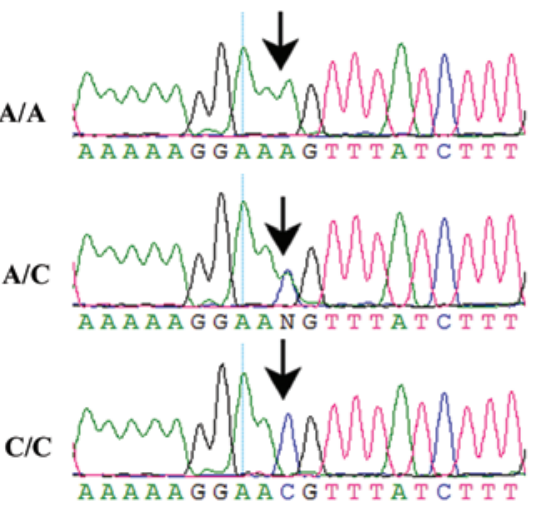

B

rs60542319
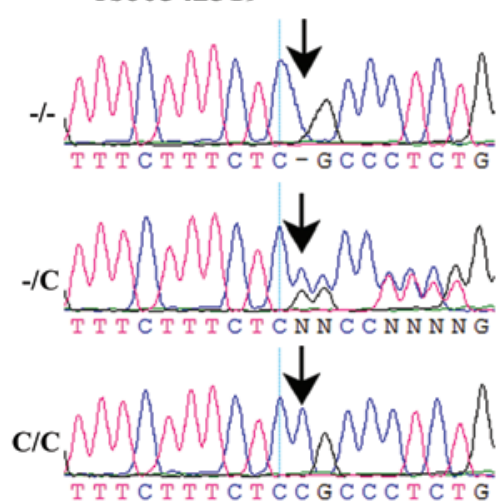

C

rs945326
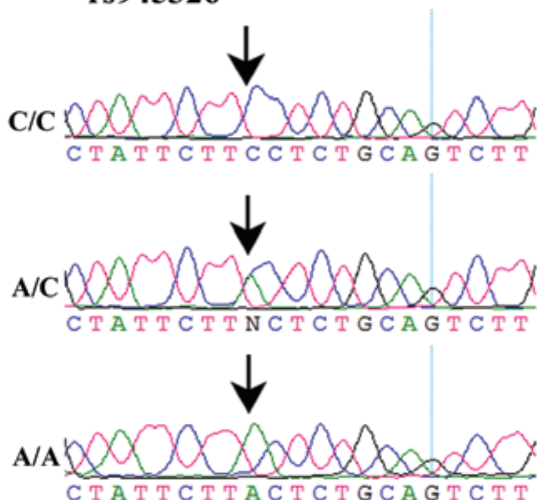

Figure 2. Sequence analysis of three SNPs: (A) rs1635529 (A/C), (B) rs60542319 (-/C) and (C) rs954326 (A/C) for genotype. The different sequence resulting from the polymorphism at this point is shown by an arrow.

was approved by the Institutional Review Board of Zhongshan Ophthalmic Center.

The inclusion criteria for individuals in each group are as follows. (A) Control group: i) best unaided visual acuity of 1.0 or better; ii) bilateral refraction between $-0.50 \mathrm{D}$ and $+2.00 \mathrm{D}$ (spherical equivalent, SE); iii) no other known eye or related systemic diseases; and iv) no family history of high myopia or hyperopia. (B) High myopia group: i) best corrected visual acuity of 0.8 or better; ii) spherical refraction at each meridian at least $\leq-6.00 \mathrm{D}$; and iii) no other known eye or related systemic diseases.

SNP genotyping. Genomic DNA was prepared from venous blood. Genotyping was carried out by sequencing the fragments with SNPs. Polymerase chain reaction was used to amplify a 561-bp DNA fragment encompassing five SNPs of interest in intron 1 of COL2Al (NCBI human genome build 36.3, NC_000012.11 for genomic DNA and NM_001844.4 for mRNA), including rs1635529,rs60542319,rs1635530,rs1635531 and rs954326 [http://www.ncbi.nlm.nih.gov/snp/, National
Center for Biotechnology Information (NCBI), Bethesda, MD, USA], using a pair of primers (Table I). Sequences of the amplicons were determined by Sanger dideoxy sequencing with ABI BigDye Terminator cycle sequencing kit version 3.1 (Applied Biosystems, Foster City, CA, USA) on an ABI 3100 Genetic Analyzer (Applied Biosystems). Sequencing results from patients as well as $C O L 2 A 1$ consensus sequences from the NCBI Human Genome Database (NC_000005.8) were imported into the SeqManII program of the Lasergene package (DNAStar Inc., Madison, WI, USA) and then aligned to identify the genotyping at each site with a polymorphism.

Statistical analysis. Initially, Hardy-Weinberg equilibrium was tested by the Chi-square test ( $\mathrm{p}$-values $<0.05$ were considered significant). Haplotype estimation and inference was determined using PHASE $(29,30)$ (PHASE software version 2; Dr Matthew Stephens, University of Chicago, Chicago, fIL, USA). Distribution of genotype, allele frequency and haplotype in the high myopia group were compared with that in the control group by using the Chi-square test. $\mathrm{P}<0.05$ was used as 


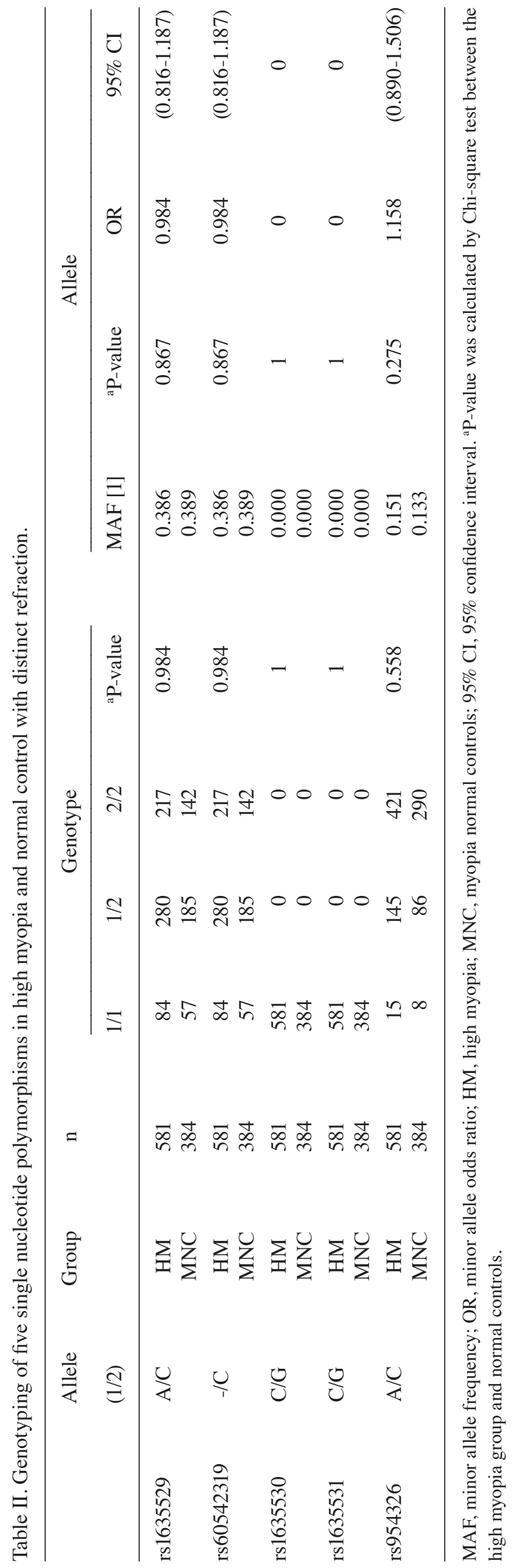

the basic statistical significance based on previous reports. In addition, the minor allele frequency (MAF), minor allele odds ratio (OR) and its $95 \%$ confidence interval (95\% CI) were calculated to estimate the effect size of the minor allele on high myopia. The statistical analysis was performed by SPSS 16.0 (SPSS Science, Chicago, IL, USA).

\section{Results}

Five SNPs: rs1635529 (A/C), rs60542319 (-/C), rs1635530 $(\mathrm{C} / \mathrm{G}), \mathrm{rs} 1635531(\mathrm{C} / \mathrm{G})$ and rs954326 (A/C) (Fig. 1) were selected for the association study, for their localization within COL2Al intron 1 using the HapMap database (http://www. ncbi.nlm.nih.gov/SNP/ provided in the public domain by the NCBI, Bethesda, MD, USA).

The genotype for each SNP was identified by direct sequencing of all subjects. Genotype and allele frequencies in the myopia and control groups are shown in Table II. Polymorphisms for rs1635530 and rs1635531 were not detected, as the $\mathrm{C}$ allele was present in all 965 subjects recruited. The other three SNPs (rs1635529 A/C, rs60542319 -/C and rs945326 $\mathrm{A} / \mathrm{C}$ ) revealed three types of genotypes, respectively (Fig. 2). There was no significant deviation from HWE for these three SNP distributions in the case and controls (data not shown).

In all high myopia subjects $(n=581)$, allele diversity of rs1635529 (allele A: 448/1162=0.39 and allele $\mathrm{C}=0.61$ ) and rs954326 (allele A: 175/11162 $=0.15$ and allele $\mathrm{C}=0.85$ ) were confirmed to HapMap data (http://www.ncbi.nlm.nih.gov/ $\mathrm{SNP} /$ ). In this study, we provide allele diversity data for rs60542319 (allele -: 448/1162=0.39 and allele $\mathrm{C}=0.61$ ) in a Han Chinese cohort, while only data for Western and Northern Europeans are listed.

For total myopia cases $(n=581)$, compared with the control group ( $n=384)$, there was no significant distribution difference for genotype, allele (Table II) and haplotype (rs1635529, rs60542319 and rs954326) frequencies (Table III).

\section{Discussion}

In the present study, SNP rs1635529, from previous reports $(23,28)$, and 4 more adjacent SNPs in COL2Al were selected to evaluate the possible relevance to high myopia. In the current study, a Chinese high myopia population composed of 581 subjects (spherical equivalent $\leq-6.00 \mathrm{D}$ ) and 384 matched controls $(-0.50 \mathrm{D} \leq$ spherical equivalent $\leq+2.00 \mathrm{D})$ participated in this association study. Our results found that no association was present between high myopia and COL2A1 SNP rs1635529, rs60542319 and rs954326. The genotype, allele and haplotype frequencies were not significantly different $(\mathrm{P}>0.05)$. It is the first report in a Chinese population to identify an association of rs1635529 with high myopia and obtain a negative result.

To date, there are two positive association results of rs1635529 with myopia, both in a Caucasian cohort. One study showed highly significant over-transmission to a cohort of 342 myopes $(\leq 0.75 \mathrm{D})$ with mixed, but Caucasian-dominant, ethnicity (23). Another study identified a significant association between rs1635529 with high-grade myopia ( $\leq-5.00 \mathrm{D})$ in two independent Caucasian family datasets, Duke (146 cases) and Cardiff (130 cases) (28). However, our results here presented negative results in the Chinese myopia group. Combined with 
Table III. Haplotype (rs1635529, rs60542319 and rs954326) frequency of COL2A1 between high myopia cases and controls.

\begin{tabular}{lcccccr}
\hline Group & $\mathrm{n}$ & \multicolumn{4}{c}{ Haplotype $^{\mathrm{a}}$} \\
\cline { 3 - 7 } & & A-C & A-A & CCC & CCA & bP-value \\
\hline HM & 581 & 449 & 0 & 538 & 175 & 0.367 \\
MNC & 384 & 298 & 1 & 369 & 100 &
\end{tabular}

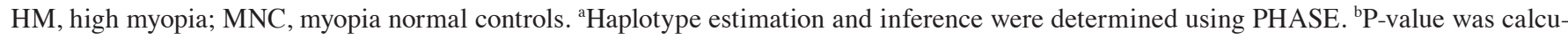
lated by the Chi-square test between the high myopia and normal control.

no association of $C O L 2 A 1$ with myopia in a Hong Kong Chinese population (130 nuclear families, $\leq-0.50 \mathrm{D})$ after four $C O L 2 \mathrm{Al}$ SNPs (in exons 1, 7, 34 and 53) were genotyped (31), COL2AI does not associate with myopia in the Chinese cohort. This may be due to the ethnic difference and more confirmation will be required in different cohorts. In addition, no linkage study has reported evidence of a strong linkage between COL2Al and high or common myopia to date, (32), other than one study with a suggestive linkage result to the COL2A1 locus on 12q, as mentioned in the same Duke and Cardiff cohort (28). It is possible that it may be due to a shared linkage disequilibrium pattern with a causative variant in a nearby gene (2).

Numerous studies have strongly implicated the role of certain genetic factors in myopia development. Association studies are widely applied to explore possible factors that can play a role in myopia formation, since its mechanism of pathogenesis remains to be clarified. COL2A1 is responsible for certain cases of Stickler Syndrome. To date, a number of studies have attempted to identify an association between non-syndromic high myopia with common SNPs in genes responsible for syndromic high myopia but, unfortunately, the results are inconclusive and generally controversial (33).

In conclusion, our study does not support an association of high myopia with rs1635529 and other SNPs in COL2A1, and further studies are required to identify candidate genes responsible for myopia.

\section{Acknowledgements}

The authors thank all of the patients and family members for their participation. This study was supported in part by the National Science Fund for Distinguished Young Scholars (30725044 to Qingjong Zhang).

\section{References}

1. Dunaway D and Berger I: Worldwide distribution of visual refractive errors and what to expect at a particular location. presentation to the International Society for Gengraphic and Epidemiologic Ophthalmology. In FOCUS Center for Primary Eye Care Development. http://www.infocusonline.org/ WORLDWIDE\%20DISTRIBUTION\%20OF\%20VISUAL\%20 REFRACTIVE\%20ERROR1.doc., 2007.

2. Yang Y, Li X, Yan N, Cai S and Liu X: Myopia: a collagen disease? Med Hypotheses 73: 485-487, 2009.

3. Wu HM, Seet B, Yap EP, Saw SM, Lim TH and Chia KS: Does education explain ethnic differences in myopia prevalence? A population-based study of young adult males in Singapore. Optom Vis Sci 78: 234-239, 2001.
4. Marr JE, Halliwell-Ewen J, Fisher B, Soler L and Ainsworth JR: Associations of high myopia in childhood. Eye 15: 70-74, 2001.

5. Fredrick DR: Myopia. Br Med J 324: 1195-1199, 2002.

6. Perez-Inigo MA, Gonzalez I, Mayoral F, Ferrer C and Honrubia FM: [Comparative study of refractive errors in simple congenital myogenic ptosis and control children]. Arch Soc Esp Oftalmol 83: 601-606, 2008 (In Spanish).

7. Akbarzadeh S and Samavati M: The comparative study of refractive errors between carpet weavers and blue collar workers. Saudi Med J 27: 912-914, 2006.

8. Zhan MZ, Saw SM, Hong RZ, et al: Refractive errors in Singapore and Xiamen, China - a comparative study in school children aged 6 to 7 years. Optom Vis Sci 77: 302-308, 2000.

9. Chandran S: Comparative study of refractive errors in West Malaysia. Br J Ophthalmol 56: 492-495, 1972.

10. Ditmars DL: A comparative study of refractive errors of young myopes and their parents. Am J Optom Arch Am Acad Optom 44: 448-451, 1967.

11. Paluru P, Ronan SM, Heon E, et al.: New locus for autosomal dominant high myopia maps to the long arm of chromosome 17. Invest Ophthalmol Vis Sci 44: 1830-1836, 2003.

12. Pararajasegaram R: VISION 2020 - the right to sight: from strategies to action. Am J Ophthalmol 128: 359-360, 1999.

13. Berntsen DA, Mutti DO and Zadnik K: Study of Theories about Myopia Progression (STAMP) design and baseline data. Optom Vis Sci 87: 823-832.

14. Rutar T and Demer JL: 'Heavy Eye' syndrome in the absence of high myopia: A connective tissue degeneration in elderly strabismic patients. J AAPOS 13: 36-44, 2009.

15. Crewther SG, Barutchu A, Murphy MJ and Crewther DP: Low frequency temporal modulation of light promotes a myopic shift in refractive compensation to all spectacle lenses. Exp Eye Res 83: 322-328, 2006.

16. Schwahn HN, Kaymak H and Schaeffel F: Effects of atropine on refractive development, dopamine release, and slow retinal potentials in the chick. Vis Neurosci 17: 165-176, 2000.

17. Flitcroft DI: A model of the contribution of oculomotor and optical factors to emmetropization and myopia. Vision Res 38: 2869-2879, 1998.

18. Miller EM: Reported myopia in opposite sex twins: a hormonal hypothesis. Optom Vis Sci 72: 34-36, 1995.

19. Wang P, Li S, Xiao X, et al: High myopia is not associated with the SNPs in the TGIF, lumican, TGFB1, and HGF genes. Invest Ophthalmol Vis Sci 50: 1546-1551, 2009.

20. Pertile KK, Schache M, Islam FM, et al: Assessment of TGIF as a candidate gene for myopia. Invest Ophthalmol Vis Sci 49: 49-54, 2008.

21. Li T, Xiao X, Li S, Xing Y, Guo X and Zhang Q: Evaluation of EGR1 as a candidate gene for high myopia. Mol Vis 14: 1309-1312, 2008.

22. Schache M, Richardson AJ, Pertile KK, Dirani M, Scurrah K and Baird PN: Genetic mapping of myopia susceptibility loci. Invest Ophthalmol Vis Sci 48: 4924-4929, 2007.

23. Mutti DO, Cooper ME, O'Brien S, et al: Candidate gene and locus analysis of myopia. Mol Vis 13: 1012-1019, 2007.

24. Hayashi T, Inoko H, Nishizaki R, Ohno S and Mizuki N: Exclusion of transforming growth factor-betal as a candidate gene for myopia in the Japanese. Jpn J Ophthalmol 51: 96-99, 2007.

25. Zhou J and Young TL: Evaluation of Lipin 2 as a candidate gene for autosomal dominant 1 high-grade myopia. Gene 352: 10-19, 2005. 
26. Tang WC, Yap MK, Yip SP: A review of current approaches to identifying human genes involved in myopia. Clin Exp Optom 91: 4-22, 2008.

27. Francomano CA, Liberfarb RM, Hirose T, et al: The Stickler syndrome is closely linked to COL2A1, the structural gene for type II collagen. Pathol Immunopathol Res 7: 104-106, 1988.

28. Metlapally R, Li YJ, Tran-Viet KN, et al: COL1A1 and COL2A1 genes and myopia susceptibility: evidence of association and suggestive linkage to the $C O L 2 A 1$ locus. Invest Ophthalmol Vis Sci 50: 4080-4086, 2009.

29. Stephens M and Donnelly P: A comparison of bayesian methods for haplotype reconstruction from population genotype data. Am J Hum Genet 73: 1162-1169, 2003.

30. Stephens M, Smith NJ and Donnelly P: A new statistical method for haplotype reconstruction from population data. Am J Hum Genet 68: 978-989, 2001.
31. Tang W, Yip S, Yap M, et al: Testing for association between COL2A1 and myopia susceptibility in Hong Kong Chinese population. Invest Ophthalmol Vis Sci. 45: Abs. 3724, 2004.

32. Andrew T, Maniatis N, Carbonaro F, et al: Identification and replication of three novel myopia common susceptibility gene loci on chromosome 3q26 using linkage and linkage disequilibrium mapping. PLoS Genet 4: e1000220, 2008.

33. Xu X, Li S, Xiao X, Wang P, Guo X and Zhang Q: Sequence variations of GRM6 in patients with high myopia. Mol Vis 15: 2094-2100, 2009. 